\title{
Paper Markov Decision Process based Model for Performance Analysis an Intrusion Detection System in IoT Networks
}

\author{
Gauri Kalnoor and Gowrishankar S \\ BMS College of Engineering, Bangalore, India
}

https://doi.org/10.26636/jtit.2021.151221

\begin{abstract}
In this paper, a new reinforcement learning intrusion detection system is developed for IoT networks incorporated with WSNs. A research is carried out and the proposed model RL-IDS plot is shown, where the detection rate is improved. The outcome shows a decrease in false alarm rates and is compared with the current methodologies. Computational analysis is performed, and then the results are compared with the current methodologies, i.e. distributed denial of service (DDoS) attack. The performance of the network is estimated based on security and other metrics.
\end{abstract}

Keywords-DDoS, intrusion detection, IoT, machine learning, Markov decision process (MDP), Q-learning, NSL-KDD, reinforcement-learning.

\section{Introduction}

The technology of the Internet of Things (IoT) is relatively new, it connects the Internet to the low hardware resources devices and then susceptible to the various malicious attach, i.e. denial of service (DOS) [1], [2]. The network-based IoT is considered to be one the fastest evolving areas, having 50 billion gadgets connected among them [3], and then vulnerable to security abuse. For example, Mirai is one of the unusual types of a botnet which triggers a largescale attack like distributed denial-of-service (DDoS) and thus strikes by mistreating some of the IoT devices [4], and even infects the CCTV IP cameras [5].

The safety of IoT is constantly improved [6]. Many frameworks and methods are developed to mitigate most network attacks. The logs with recorded abuse historical data are observed, based on methods using machine learning which can reach a large network - up to millions in a day.

The intrusion detection system (IDS) is an essential component in the security of the network to protect the target network which comprises of irregular actions and threats during interruption of network traffic. Thus, there is a separation of normal activity and anomalous activity in the network. A comprehensive IDS group can be obtained in two classes. Misuse-based IDS is the interrupt that notices the known strategies. The limit of the primary technique to anticipate new and obscure assaults is restricted. The signature-based IDS is dependent on the irregularity identification and works by making a profile of ordinary conduct of the network, then later recognizing it as any anomalous conduct [3].

In the proposed work, an artificial intelligence (AI) based algorithm has been proposed for developing an IDS for detection of malicious attacks and also monitors the data streams generated from IoT and WSNs [6].

It is an enhanced method of Markov decision process with Q-Network algorithm which gives an optimal best solution in terms of performance of IoT networks. Thus, it is an important and challenging issue to be considered, and decision modeling is applied to obtain the optimal solution. The main contributions of this article are summarized below:

- the RL-based IDS is proposed by exploiting the extended Markov decision process (MDP) algorithm,

- the RL calculation is consolidated on IDS (RL-IDS) with the end goal that the survey for cases like a basic foundation is obtained by unique digital-based hazards for IoT and WSN continuously,

- a Q-network is applied with the end goal that the assessment of Q-work is recognized by conveying IDS into RL. A few tests are performed for the assessment of the execution of the proposed model in the environment considered.

The remaining sections of this paper are presented as follows. Section 2 describes the related work. Section 3 introduces the method for security and reinforcement learning. In Section 4, the system model is formulated and RL-IDS methodology is described. In Section 5, performance is investigated and results are presented. In Section 5, the evaluation carried out for the proposed RL-based IDS scheme is explained and then compared in Section 6 with supervised machine learning schemes. Lastly, the work concludes with the experiments and analysis in Section 7. 


\section{Related Work}

In recent works, many authors have applied standard techniques of machine learning (ML), such as principal component analysis (PCA) and linear discriminant analysis (LDA), as these classification-based algorithms can detect normal records with high precision and identify the abnormal records such that the performance of an IDS can be managed [7]-[11]. In [12], the authors have proposed deep feature embedding to reduce the size or magnitude of data from the network based on IoT in a real-time application by considering the "edge of deep learning". Likewise, in [13] the preprepared worldview is applied such that the identification and quickness are helped with traditional ML-based calculations.

In [14], the authors have observed that the IoT technology makes possible to connect different smart objects, through the Internet. The authors have formulated a novel QoS management schemes based on power control algorithm. The unexplored R-learning algorithm is used as a docitive paradigm by the authors where the system agents teach other agents to adjust the power levels, thus reducing the complexity in computation and increasing speed in the learning process.

In [15] the optimization has been incorporated into an MDP which can minimize the evaluation metric as long-term average delay. The continuity of state and action space due to the high dimensionality is considered by the author where deep reinforcement learning based dynamic resource management (DDRM) algorithm is proposed. This enables the joint optimization with computing resource and transmission power. The authors have compared the simulated results with conventional URM, RRM and A3C algorithms mainly which reduces the delay in task effectively.

Also, taking as an illustration of the idea-based IDS, Qlearning of reinforcement learning (RL) has been investigated thoroughly by examining and protecting the sensor network that utilizes the dynamic methodology and ideal activities based on the arrangement of states in the respective IoT environment [16]. There are numerous papers on scientific classification, position, and the ML current advancements in data security, i.e. [17], [18]. Structured [19] ML techniques have been applied to location interruption for network information. The exemplary ML models applied to IDS were: support vector machine (SVM), multilayer perceptron (MLP), k-nearest neighbors (KNN), decision trees (DT), naive Bayes (NB), and random forest.

\section{Security in IoT}

To meet the ideal security necessities, a complete perspective on network security is required. The accompanying key security properties ought to be viewed when building up a convincing IoT security methodology.

- confidentiality - it is a crucial security standard for IoT structures. IoT devices can store and move sensi- tive information that shouldn't be wrongly found by individuals [21],

- authentication - the verification of both communication parties must be completed before performing other procedures,

- integrity - the IoT applications need the legitimate constituents to be uniquely altered where the information is moved through the remote correspondence,

- availability - the authorized users should be consistently able to access the IoT network,

- authorization - this includes granting privileges to clients for an IoT structure [22],

\subsection{Reinforcement Learning-based IDS}

Beginning by characterizing the idea of RL, and other augmentation of ML dependent on Markov decision process (MDP), first a reward function $R$ is defined providing state $s$ to IDS. It is characterized with five IDS concepts as below. System state space. The arrangement of states gained by the IDS is $S=s_{0}-$ ordinary, $s_{1}-$ identification, $s_{2}-$ no detection, where $s_{0}$ demonstrates the typical traffic record in the WSN record, $s_{1}$ implies the location of IDS assaults on traffic, and $s_{2}$ demonstrates that IDS can't recognize assaults.

Action space. A set of possible actions that the IDS can perform, can be expressed by:

$$
A=\left\{a_{0}, a_{1}, a_{2}, a_{3}, \ldots, a_{m}\right\}
$$

where $a_{k}$ indicates the type of IDS reaction in the $k$-th attack class and $k=0,1,2, \ldots, m, p$, for example, according to Table 1. The shares are sorted according to their risk level: $a_{0}<a_{1}<a_{2}<a_{3}<\ldots<a_{m}$.

Table 1

Known attacks and their risk level

\begin{tabular}{|c|c|}
\hline Risk & Attack instances \\
\hline Low & Gues-passwd, Warezclient, FTP-write \\
\hline Medium & Satan, Portsweep, Nmap \\
\hline High & DNS-poisoning, Cross-site-scripting (XSS), \\
& ARP-spoofing \\
\hline Critical & ICMP flood, Land, Smurf, Ping of death, Apache 2 \\
\hline
\end{tabular}

Reward function. The rewarded function is negative when the IDS makes the best move to secure the framework regardless of whether the scheme against the activity is too costly, and positive when the IDS chooses the right activity. 
The estimation of the reward is:

$$
\left\{\begin{aligned}
R_{p} & \text { for } \left.s_{t}=0 \text { and } a_{t}=a_{0}, s_{t}\right)= \\
1-\mu_{j}\left(a_{t}\right) R_{p} & \text { for } s_{t}=s_{0} \text { and } a_{t} \in\left\{a_{1}, \ldots, a_{m}\right\} \\
R_{p} & \text { for } s_{t}=s_{1} \text { and } a_{t}=a_{k} \\
1-\lambda_{j}\left(a_{t}\right) R_{p} & \text { for } s_{t}=s_{1} \text { and } a_{t} \in\left\{a_{0}, \ldots, a_{k-1}\right\} \\
R_{n} & \text { for } s_{t}=s_{1} \text { and } a_{t} \in\left\{a_{k+1}, \ldots, a_{m}\right\} \\
R_{p} & \text { for } s_{t}=s_{2} \text { and } a_{t}=a_{m}
\end{aligned}\right\},
$$

where $0<u_{j}\left(a_{j}\right)<1,0<\lambda_{j}\left(a_{t}\right)<1$ and $0<\theta_{j}\left(a_{t}\right)<1$. The $r_{t}$ refer to the reward $s_{t}$ is the state of the sensor node, $a_{t}$ is the action of the sensor at $t$ time.

The reward in each time $t$ is:

$$
r_{t}\left(S_{t}=s, a_{t}=a\right)=\Sigma_{s^{\prime} \in S} P\left(\frac{s}{s^{\prime}}, a\right) r_{t}\left(s^{\prime}, a\right)
$$

State transition probability. The transition probability matrix at time $t$ for $a \in \mathrm{A}$ is:

$$
\mathbf{P}_{\mathbf{a}}=\left[\begin{array}{lll}
\beta_{1,1}^{a} & \beta_{1,2}^{a} & \beta_{1,3}^{a} \\
\beta_{2,1}^{a} & \beta_{2,2}^{a} & \beta_{2,3}^{a} \\
\beta_{3,1}^{a} & \beta_{3,2}^{a} & \beta_{3,3}^{a}
\end{array}\right] .
$$

Given by $\beta^{a}$ :

$$
\begin{array}{r}
i, j=p\left(s_{t}+\frac{1}{s_{t}}\right)=p\left(\frac{s_{i}}{s_{j}}, a\right) \text { for } i, j=1,2,3 . \\
\Sigma_{j=1}^{3} \beta_{i, j}^{a}=1, i=1,2,3 \text { and } a \in A .
\end{array}
$$

Discount factor. $0<\gamma<1$. The IDS arbitrarily choose $a_{t}$, and the environment samples the reward $r_{t}\left(s_{t}, a_{t}\right)$ according to the state of arrival $s$. The agent then receives an incentive in the following state $s_{t+1}$. Besides, $\pi$ is a specific policy from $s_{t}$ to $s_{t+1}$ specifying $a_{t}$ retrieved in each state $s_{t}$. Then, the strategy is updated to generate sample paths $\left(s_{0}, a_{0}, r_{0}\right)$, $\left(s_{1}, a_{1}, r_{1}\right),\left(s_{2}, a_{2}, r_{2}\right) \ldots$ Let us define $\pi=\left(\pi_{1}, \pi_{2}, \ldots\right)$ as the best policy vector. The goal of the data stream is to get $\pi_{t}$, which represents the best pattern based on system status. Therefore, the expected maximum sum of IDS rewards at $t$, is given by:

$$
\pi^{*}=\underset{a \in A}{\arg \max }\left[r_{t}\left(s_{t}, a_{t}\right)+\Sigma_{s^{\prime} \in S} P_{t}\left(s^{\prime} \mid s, a\right) V_{I-1-t}\left(s^{\prime}\right)\right] .
$$

The optimal value function $V_{i+1}$ defines the IDS which can be chosen as the best state. It can be found out from each phase:

$$
\begin{gathered}
V_{i+1}(s)=\underset{a \in A}{\arg \max }\left[r_{I-1-t}\left(s_{t}, a_{t}\right)+\right. \\
\left.\Sigma_{s^{\prime} \in S} P_{I-1-t}\left(s^{\prime} \mid s, a\right) V_{i}\left(s^{\prime}\right)\right] .
\end{gathered}
$$

Next, the timestamp size is determined, using the concept of Q-learning. In every state, the best action $a$ is chosen and the algorithm Q-learning applied, so that the updates can be performed. The optimal policy $\pi *$ is calculated according to the best action. If there are no optimal actions found, then the learning samples $0<\alpha<1$ are applied.

$$
\begin{array}{r}
Q\left(s_{t}-a_{t}\right)=Q\left(s_{t}, a_{t}\right)+ \\
\alpha\left[r_{t}+\gamma \max _{a t} \in A Q\left(s_{t+1}, a_{t}\right)-Q\left(s_{t}, a_{t}\right)\right] .
\end{array}
$$

The pair $(s, a)$ is updated to determine the step having the best reward. In each iteration, the prediction of IDS has state value function $V_{i+1}$ and then a Q-table is constructed by using Q-learning, where the lines signify the columns and states $s$ representing the actions $a$. In each state $s_{t}$, the reward $r_{t}$ is observed corresponding to an action $a_{t}$ realized by the agent. The action at the next state $\left(s_{t+1}\right)$ is also observed in [21], and the approximate value of $Q$ is updated to satisfy the Bellman equation:

$$
\begin{array}{r}
Q\left(s_{t+1}-a_{t+1}\right)=(1-\alpha) Q\left(s_{t}, a_{t}\right)+ \\
\alpha\left[r_{t}+\gamma \max _{a^{\prime}} \in A Q\left(s^{\prime}, a^{\prime}\right)\right] .
\end{array}
$$

\section{Proposed Model}

The random forests (RF) algorithm is used to classify a large amount of data. Several algorithms like decision trees and merging trees are used during classification to train the sample data available. The final output during classification chooses the most selected class [7].

In this section, the details of the deployment of the Qlearning network-based model are provided aiming to monitor and predict the cyber-attacks in critical infrastructures of sensed big data streams. The discussion is encompassed in the following aspects:

- the attack risks and their different degree,

- the pre-processing details engaged to clean data and filter,

- the strategy of the interaction of IDS model by the agent to secure the attacks,

- the Q-function estimation and its results by considering the best decision.

The architecture of the proposed system is shown in Fig. 1, which presents the sensor data of WSN and the RL-IDS mechanism requested to make a decision.

At pre-processing stage, the network traffic is registered for every type of attack and then invalid and redundant records are removed. Next, the transformation of the record is done based on the type of attack [9]. At the first step, data aggregation obtained by the sensor [20] is performed so that the data volume is reduced.

Next, the Q-network (QN) is applied by using the Qfunction for estimation of best action to the attack. It improves the prediction and the estimation of action values effectively among the state's set by applying the non-linear function: $Q\left(s_{t}, a_{t} ; \theta\right) \approx Q\left(s_{t+1}, a_{t+1}\right)$.

The $\theta$ represents neuron weights to be changed by the end of each iterative step $i$. The implementation of Q-network is further improved by:

- utilizing a step forward for the present state $s$ to get predictive $\mathrm{Q}$ values,

- applying the replay (like historical IDS for the interactive process) into data let $H t=$ 


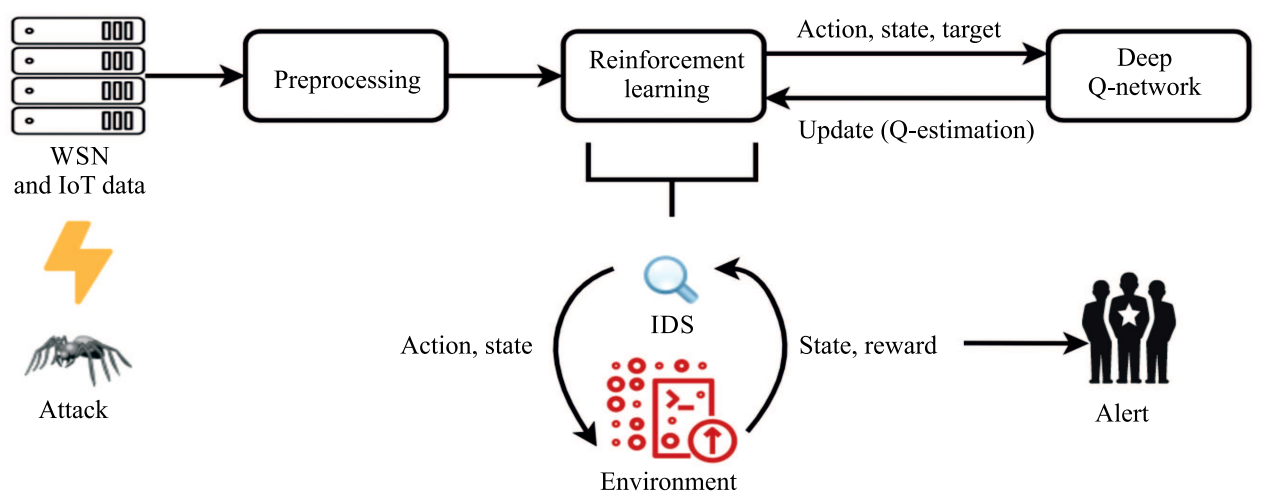

Fig. 1. Proposed method of improval the IoT and WSN based RL-IDS.

$\{h(1), h(2), \ldots, h(t)\}$ within an over-time $t$ as $f_{t}=$ $\left(s_{t}, a_{t}, r_{t}, s_{t+1}\right)$,

- updating the Q-network based on the data from training $(r, s, a, s)$ over the target Q-value with optimization of the loss-function during an iterative step noted as:

$$
\begin{gathered}
L_{i}\left(\theta_{i}\right)=E\left\{\left[x_{i}-Q\left(s, a, \theta_{i}\right)\right]^{2}\right\}, \\
x_{i}=r_{t}+\gamma \arg \max a^{\prime} Q\left(s^{\prime}, a^{\prime}, \theta_{i-1}\right),
\end{gathered}
$$

- applying back-propagation with loss function's gradient, the weights are updated corresponding to the $\theta$ parameters as:

$$
\nabla_{\theta_{i}} L_{i}\left(\theta_{i}\right)=E\left\{\left[x_{1}-Q\left(s, a, \theta_{i}\right)\right] \nabla_{\theta_{i}} Q\left(s, a, \theta_{i}\right)\right\} .
$$

\subsection{Model Description}

In the proposed scheme, the problem for QoS control is tackled based on the approach of R-learning algorithm. The main aim of every QoS scheduler is maximization the amount of data transmitted with low power consumption. For this fundamental trade-off, the function $U$ is defined to analyze the ratio of throughput to power. Thus, the function for QoS scheduler at $i$-th position $U_{i}$ is:

$$
\begin{array}{r}
U_{i}\left(B_{j}^{i}, B_{-i}\right)=\frac{T S_{i}(B)}{B_{j}^{i}}, \text { s.t., } B_{j}^{i} \in B_{i}, B= \\
\quad I I_{i \varepsilon N} B_{i} \mid B_{i} \in\left[B_{1}^{i}, B_{m}^{i}\right],
\end{array}
$$

where $B_{-i}$ is the transmit power vector without $B_{i}$, and $T S_{i}(B)$ is the throughput scheduler.

In wireless communication, the signal to interference noise ratio (SINR) in the given effective range $\gamma_{i}$ is measured while computing the throughput at $i$-th scheduler $T S_{i}$ and can be expressed using:

$$
T S_{i}(B)=W \cdot \log _{2}\left(1+\frac{\gamma_{i}(A)}{\Omega}\right)
$$

where $W$ is referred to as bandwidth of the channel assigned in through IoT network, $\Omega(\Omega \geq 1)$ is the gap between capacity and the uncoded M-ary quadrature amplitude modulation (M-QAM).

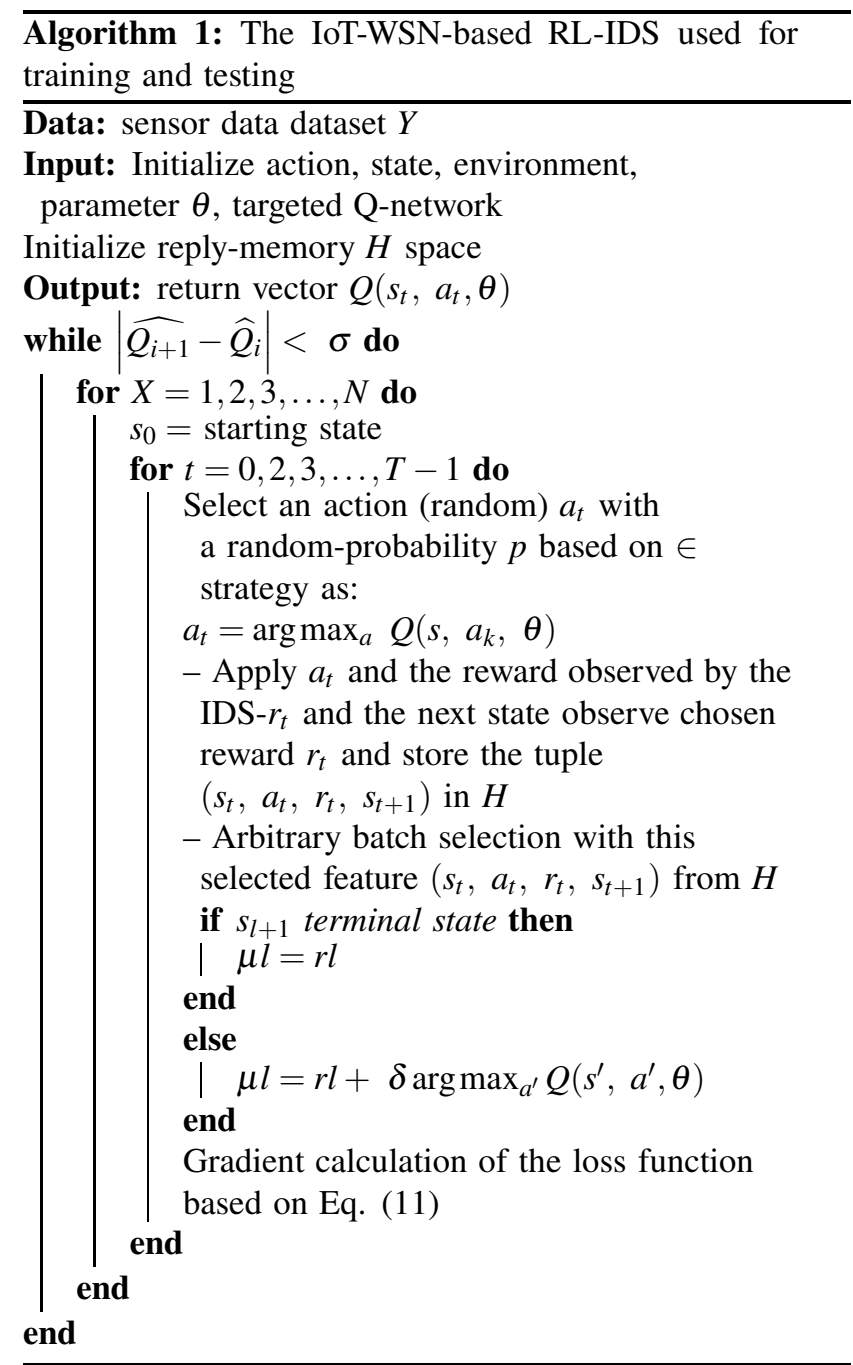


Table 2

Dataset used for evaluation

\begin{tabular}{|c|c|c|c|c|}
\hline Category & Port & Attack & Tools & Size [bytes] \\
\hline Information collect & & $\begin{array}{l}\text { Scanning of service } \\
\text { OS fingerprinting }\end{array}$ & $\begin{array}{l}\text { Nmap, hping3, } \\
\text { xprobe2 Nmap }\end{array}$ & $\begin{array}{l}1.4 \mathrm{MB} \\
358 \mathrm{~KB}\end{array}$ \\
\hline \multirow{2}{*}{ Denial of service } & UDP, ТCP НTTP & Distributed DoS & $\begin{array}{c}\text { hping3 } \\
\text { golden-eye } \\
\text { hping3 }\end{array}$ & $\begin{array}{r}19.5 \mathrm{MB} \\
18.8 \mathrm{MB} \\
19.7 \mathrm{~KB} \\
\end{array}$ \\
\hline & TCP, HTTP UDP & DoS & $\begin{array}{c}\text { hping3 } \\
\text { hping3 } \\
\text { golden-eye }\end{array}$ & $\begin{array}{c}11.2 \mathrm{MB} \\
21.7 \mathrm{MB} \\
29.7 \mathrm{~KB}\end{array}$ \\
\hline Information theft & & $\begin{array}{l}\text { Key-logging } \\
\text { data theft }\end{array}$ & $\begin{array}{l}\text { Metasploit } \\
\text { Metasploit }\end{array}$ & $\begin{array}{r}1369 \\
118\end{array}$ \\
\hline
\end{tabular}

The environment was made by consolidating traffic and Table 2 shows the used datasets and software tools.

\section{Evaluation Criteria}

The validation of proposed algorithm is researched by two measures:

- Accuracy - this metric is measured as the degree of closeness between the actual and the predicted value,

- Precision - this is a metric that describes the accuracy level obtained from the mentioned information and the outcomes anticipated by the executed model. Consequently, accuracy is the proportion of true positive forecasts contrasted with general aftereffects of positive expectation.

Table 3 shows the boundaries or limits used for CNN and MLP algorithms.

Table 3

Parameters of algorithms used for testing

\begin{tabular}{|c|c|c|c|c|}
\hline Algorithm & $\begin{array}{c}\text { Batch } \\
\text { size }\end{array}$ & $\begin{array}{c}\text { Function } \\
\text { (activation) }\end{array}$ & Optimizer & Epochs \\
\hline $\begin{array}{c}\text { Convolution neural } \\
\text { network (CNN) }\end{array}$ & $32,64,128$ & $\begin{array}{c}\text { Softmax, } \\
\text { ReLu }\end{array}$ & Adam & $10,30,50$ \\
\hline $\begin{array}{c}\text { Multilayer } \\
\text { perceptron (MLP) }\end{array}$ & $32,64,128$ & $\begin{array}{c}\text { Softmax, } \\
\text { ReLu }\end{array}$ & Adam & $10,30,50$ \\
\hline $\begin{array}{c}\text { Markov decision } \\
\text { process (MDP) }\end{array}$ & $32,64,128$ & $\begin{array}{c}\text { Softmax, } \\
\text { ReLu }\end{array}$ & Adam & $10,30,50$ \\
\hline
\end{tabular}

A major drawback of any IoT sensor network is that these devices work in remote networks and have to be sustained on their battery life. Hence the average energy consumed by the device plays a vital role which depends on its performance as shown in Fig. 2, the node shows that the MDP algorithm provides a less amount of energy consumption when compared with CNN and MLP algorithm.

MDP provides significant results as false detection is reduced even when the number of nodes is increased as shown in Fig. 3. As the number of nodes increases the false detection is getting reduced as compared with MLP and CNN.

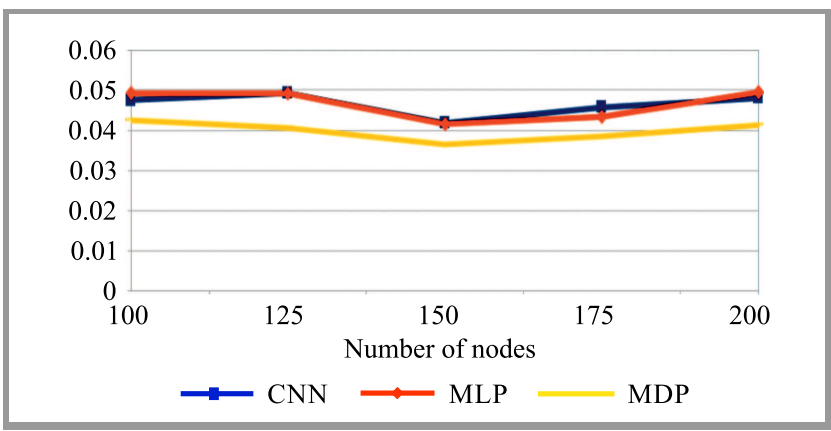

Fig. 2. Average energy consumption by number of nodes.

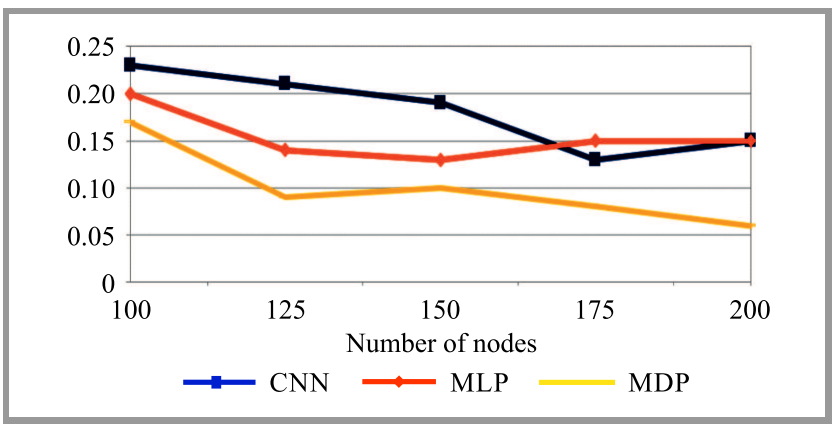

Fig. 3. False alarm rate.

The system of IoT mainly in a wireless system depends on the success rate of message delivery even when the number of nodes are increased and have a successful delivery rate which is provided in Fig. 4. In this plot all algorithm with the proposed algorithm, the throughput is given and can be observed that the MDP performance is good for throughput when nodes are more.

A comparison figure of the detection rate of IoT systems is shown in Fig. 5 which depicts that the detection rate at the receiver node in MDP is better when compared with $\mathrm{CNN}$ and MLP.

Figure 6 presents normalized overhead for several nodes in the IoT network when compared with all other algorithms with the reinforced algorithm MDP, it provides better performance for normalized overhead when compared with MLP and CNN. Parameters from Table 4 were used in this plot. 


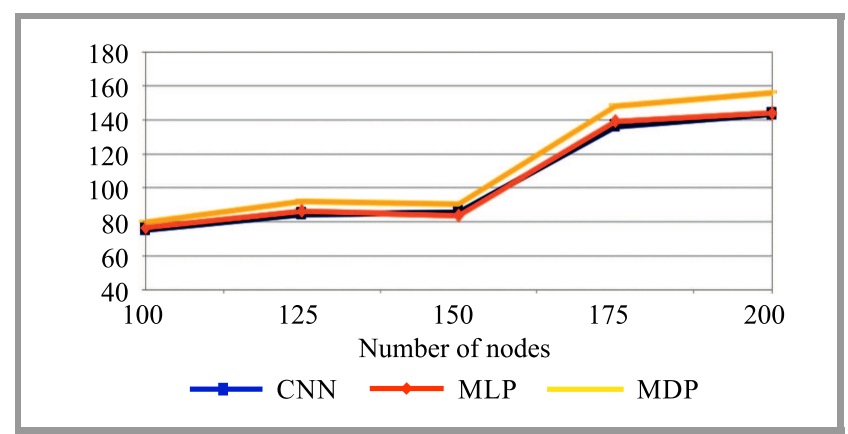

Fig. 4. Throughput rate of change.

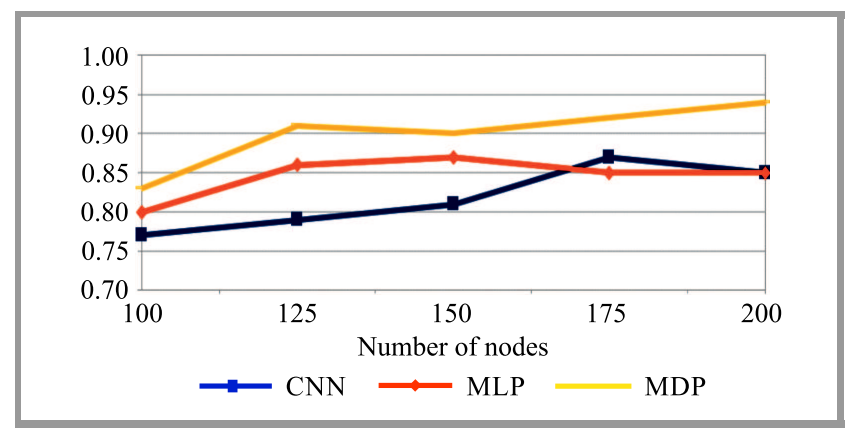

Fig. 5. Detection rate.

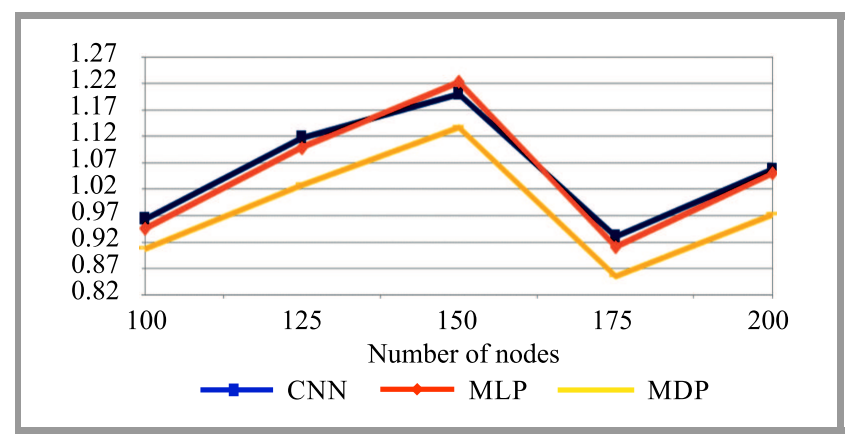

Fig. 6. Normalized overhead.

Table 4

Evaluation metrics (detection rate of attacks)

\begin{tabular}{|c|c|c|c|c|c|}
\hline \multirow{2}{*}{ Algorithm } & \multicolumn{5}{|c|}{ Metrics } \\
\cline { 2 - 6 } & $\begin{array}{c}\text { DDoS } \\
\text { attack }\end{array}$ & $\begin{array}{c}\text { DoS } \\
\text { attack }\end{array}$ & Reconnaissance & $\begin{array}{c}\text { Normal } \\
\text { (AUC) }\end{array}$ & $\begin{array}{c}\text { Theft } \\
\text { (AUC) }\end{array}$ \\
\hline MDP & 0.99 & 0.99 & 0.97 & 0.99 & 0.95 \\
\hline CNN & 0.98 & 0.97 & 0.98 & 0.98 & 0.99 \\
\hline MLP & 0.55 & 0.49 & 0.96 & 0.97 & 0.97 \\
\hline
\end{tabular}

Table 5 represents the classification and comparison results based on the feature selection and the AUC precision metrics.

In Table 6, the mean accuracy is expanded as the number of study ages for the MLP classifier. For CNN, there was a decrease as the quantity (in terms of numbers) of epochs increased from 10 to 50 .

Table 7 shows the same accuracy evaluation for size of 64 . For this situation, the accuracy (batch size 64) diminished
Table 5

Comparison analysis

\begin{tabular}{|c|c|c|c|}
\hline Algorithm & AUC & Precision & Sensitivity \\
\hline MDP & 0.99 & $99.80 \%$ & $98.55 \%$ \\
\hline CNN & 0.92 & $96.75 \%$ & $97.00 \%$ \\
\hline MLP & 0.89 & $95.05 \%$ & $93.02 \%$ \\
\hline
\end{tabular}

Table 6

The accuracy evaluation for batch size 32

\begin{tabular}{|c|c|c|c|}
\hline Algorithm & Epoch & Mean Accuracy & Elapsed time \\
\hline MDP & 10 & $93.22 \%$ & $60 \min 12 \mathrm{~s}$ \\
\hline CNN & 10 & $91.75 \%$ & $58 \min 39 \mathrm{~s}$ \\
\hline MLP & 10 & $54.07 \%$ & $39 \min 09 \mathrm{~s}$ \\
\hline MDP & 30 & $91.03 \%$ & $165 \min 25 \mathrm{~s}$ \\
\hline CNN & 30 & $89.72 \%$ & $158 \min 30 \mathrm{~s}$ \\
\hline MLP & 30 & $63.95 \%$ & $124 \min 33 \mathrm{~s}$ \\
\hline MDP & 50 & $90.00 \%$ & $230 \min 21 \mathrm{~s}$ \\
\hline CNN & 50 & $89.30 \%$ & $229 \min 22 \mathrm{~s}$ \\
\hline MLP & 50 & $63.00 \%$ & $186 \min 47 \mathrm{~s}$ \\
\hline
\end{tabular}

with the expansion epochs for the classifier (MLP). Data decreasing a bit while the number of epochs is increased from 10 to 50 in $\mathrm{CNN}$.

Table 7

Accuracy for batch size 64

\begin{tabular}{|c|c|c|c|}
\hline Algorithm & Epoch & Mean accuracy & Elapsed time \\
\hline MDP & 10 & $92.00 \%$ & $18 \min 40 \mathrm{~s}$ \\
\hline CNN & 10 & $91.15 \%$ & $20 \min 57 \mathrm{~s}$ \\
\hline MLP & 10 & $76.92 \%$ & $26 \min 56 \mathrm{~s}$ \\
\hline MDP & 30 & $92.30 \%$ & $62 \min 17 \mathrm{~s}$ \\
\hline CNN & 30 & $91.02 \%$ & $64 \min 18 \mathrm{~s}$ \\
\hline MLP & 30 & $54.04 \%$ & $64 \min 19 \mathrm{~s}$ \\
\hline MDP & 50 & $92.30 \%$ & $114 \min 60 \mathrm{~s}$ \\
\hline CNN & 50 & $90.64 \%$ & $112 \min 55 \mathrm{~s}$ \\
\hline MLP & 50 & $53.89 \%$ & $102 \min 20 \mathrm{~s}$ \\
\hline
\end{tabular}

Table 8 shows the outcome for block size of 128 . The normal exactness seems to increment along with the expanding number of the experiment of epochs for MLP-based classifier. For the $\mathrm{CNN}$, a slight diminishing was observed as the number of epochs rises from 10 to 30 . In all cases the larger batch size the shorter application lifetime.

\section{Conclusion}

In the proposed work, the reinforcement learning in a network is examined. The valuation of the RL-IDS model is incorporated and compared with different ML and DL algorithms such as CNN and LP. The RL calculation gave the best outcome and precision and AUC leads in multiclass 
Table 8

Mean accuracy for batch size 128

\begin{tabular}{|c|c|c|c|}
\hline Algorithm & Epoch & Mean accuracy & Elapsed time \\
\hline MDP & 10 & $92.50 \%$ & $12 \min 12 \mathrm{~s}$ \\
\hline CNN & 10 & $90.87 \%$ & $11 \min 33 \mathrm{~s}$ \\
\hline MLP & 10 & $54.10 \%$ & $10 \min 16 \mathrm{~s}$ \\
\hline MDP & 30 & $93.00 \%$ & $40 \min 50 \mathrm{~s}$ \\
\hline CNN & 30 & $90.76 \%$ & $45 \min 44 \mathrm{~s}$ \\
\hline MLP & 30 & $54.43 \%$ & $27 \min 58 \mathrm{~s}$ \\
\hline MDP & 50 & $92.03 \%$ & $55 \min 27 \mathrm{~s}$ \\
\hline CNN & 50 & $91.27 \%$ & $54 \min 27 \mathrm{~s}$ \\
\hline MLP & 50 & $79.01 \%$ & $46 \min 18 \mathrm{~s}$ \\
\hline
\end{tabular}

characterization. With epoch increase a slight reduction in precision is observed, while in the 128-batch preliminaries, there was an increase in accuracy. A double change in MLP could make the estimation cycle 1.4 to $2.6 \mathrm{~s}$ faster, while CNN could make the figuring cycle 1.8 to $2.4 \mathrm{~s}$ shorter. Later on, the models with various calculations are likely created and different calculations for AI or profound learning are joined. Moreover, this calculation ought to be actualized in NIDS so it very well may be utilized progressively to alleviate attacks.

\section{References}

[1] M. Roopak, G. Y. Tian, and J. Chambers, "Deep learning models for cyber security in IoT networks", in Proc. IEEE 9th Annual Comput. and Commun. Workshop and Conf. $(C C W C)$, Las Vegas, NV, USA, 2019, pp. 452-457 (DOI: 10.1109/CCWC.2019.8666588).

[2] X. Yuan, C. Li, and X. Li, "DeepDefense: identifying DDoS attack via deep learning", in Proc. of the 2017 IEEE Int. Conf. on Smart Comput. (SMARTCOMP), Hong Kong, China, 2017, pp. 1-8 (DOI: 10.1109/SMARTCOMP.2017.7946998)

[3] D. Evans, "The Internet of Things: how the next evolution of the Internet is changing everything", Cisco Internet Business Solutions Group (IBSG), 2011 [Online]. Available: http://www.cisco.com/ c/dam/en_us/about/ac79/docs/innov/IoT_IBSG_0411FINAL.pdf

[4] C. Kolias, G. Kambourakis, A. Stavrou, and J. Voas, "DDoS in the IoT: Mirai and other botnets", Computer, vol. 50. no. 7, 2017, pp. 80-84 (DOI: 10.1109/MC.2017.201).

[5] P. Radanliev et al., "Future developments in cyber risk assessment for the Internet of Things", Computers in Industry, vol. 102, pp. 14-22, 2018 (DOI: 10.1016/j.compind.2018.08.002).

[6] E. Bertino and N. Islam, "Botnets and Internet of Things security", Computer, vol. 50, no. 2, pp. 76-79, 2017 (DOI: 10.1109/MC.2017.62).

[7] M. A. Al-Garadi, A. Mohamed, A. Al-Ali, X. Du, and M. Guizani, "A Survey of machine and deep learning methods for Internet of Things (IoT) security", IEEE Commun. Surveys \& Tutorials, vol. 22, no. 3, pp. 1646-1685, 2020 (DOI: 10.1109/COMST.2020.2988293).

[8] A. Okwori, "Intrusion detection in Internet of Things (IoT)", Int. J. of Advanced Res. in Computer Sci., vol. 9, pp. 504-509, 2018 (DOI: 10.26483/ijarcs.v9i1.5429).

[9] Y. Meidan, "ProfilIoT: a machine learning approach for IoT device identification based on network traffic analysis", in Proc. of the Symp. on Applied Comput. - SAC '17, Marrakech, Morocco, 2017, pp. 506-509 (DOI: 10.1145/3019612.3019878).

[10] E. Anthi, L. Williams, M. Slowinska, G. Theodorakopoulos, and P. Burnap, "A Supervised intrusion detection system for smart home IoT devices", IEEE Internet of Things J., vol. 6, no. 5, 2019, pp. 9042-9053 (DOI: 10.1109/JIOT.2019.2926365).
[11] A. Azmoodeh, A. Dehghantanha, and K.-K. R. Choo, "Robust malware detection for Internet of (battlefield) things devices using deep eigenspace learning", IEEE Trans. Sustain. Comput., vol. 4, no. 1, 2019, pp. 88-95 (DOI: 10.1109/TSUSC.2018.2809665).

[12] S. Hajiheidari, K. Wakil, M. Badri, and N. J. Navimipour, "Intrusion detection systems in the Internet of Things: A comprehensive investigation", Comput. Netw., vol. 160, pp. 165-191, 2019 (DOI: 10.1016/j.comnet.2019.05.014).

[13] R. Nicolescu et al., "Mapping the values of IoT", J. Inf. Technol., vol. 33, pp. 345-360, 2019 (DOI: 10.1057/s41265-018-0054-1).

[14] S. Sheng et al., "Deep reinforcement learning-based task scheduling in IoT edge computing", Sensors (Basel), vol. 21, no. 1666, 2021 (DOI: 10.3390/s21051666).

[15] Y. Chen et al., "Deep reinforcement learning based dynamic resource management for mobile edge computing in industrial Internet of Things", IEEE Transac. on Industrial Informat., vol. 17, no. 7, pp. 4925-4934, 2021 (DOI: 10.1109/TII.2020.3028963).

[16] M. Elrawy, A. Awad, and H. Hamed, "Intrusion detection systems for IoT-based smart environments: a survey", J. Cloud Comput., vol. 7, no. 21, 2018 (DOI: 10.1186/s13677-018-0123-6).

[17] M. H. Bhuyan, D. K. Bhattacharyya, and J. K. Kalita, "Network anomaly detection: methods, systems and tools", IEEE Communications Surveys \& Tutorials, vol. 16, no. 1, pp. 303-336, 2013 (DOI: 10.1109/SURV.2013.052213.00046).

[18] F. Hussain, R. Hussain, S. A. Hassan, and E. Hossain, "Machine learning in IoT security: current solutions and future challenges", arXiv [Online]. Available: https://arxiv.org/pdf/1904.05735.pdf

[19] K. A. P. da Costa, J. P. Papa, C. de Oliveira-Lisboa, R. Munoz, and V. H. C. de Albuquerque, "Internet of Things: a survey on machine learning-based intrusion detection approaches", Computer Networks, vol. 151, pp. 147-157, 2019 (DOI: 10.1016/j.comnet.2019.01.023).

[20] Z. Chen, C. K. Yeo, B. S. Lee, and C. T. Lau, "Autoencoder-based network anomaly detection", in 2018 Wireless Telecommun. Symp. (WTS), Phoenix, AZ, USA, 2018, pp. 1-5 (DOI: 10.1109/WTS.2018.8363930).

[21] S. U. Jan, S. Ahmed, V. Shakhov, and I. Koo, "Toward a lightweight intrusion detection system for the Internet of Things", IEEE Access, vol. 7, pp. 42450-42471, 2019 (DOI: 10.1109/ACCESS.2019.2907965).

[22] M. Abomhara and G. M. Koien, "Cyber security and the Internet of Things: vulnerabilities, threats, intruders and attacks", J. of Cyber Secur. and Mobil., vol. 4, no. 1, pp. 65-88, 2015 (DOI: $10.13052 /$ jcsm2245-1439.4).

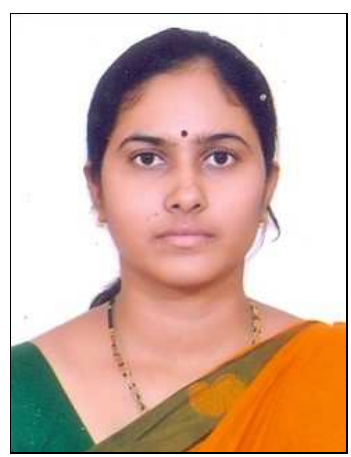

Gauri Kalnoor received her B.E. and M.Tech. from the department of Computer Science and Engineering, Viswesaraya Technological University, Belgavi in 2008 and 2010, respectively. She has worked in Central University of Karnataka as an Assistant Professor and in Wipro Technologies as a Project Engineer. She is a research scholar in B.M.S.C.E. Research Centre and her research area is Internet of Things. She is interested in coding and analysis of machine learning techniques.

(iD) https://orcid.org/0000-0001-9970-4697

E-mail: kalnoorgauri@gmail.com

B.M.S.C.E

Basavangudi

Bangalore, India 


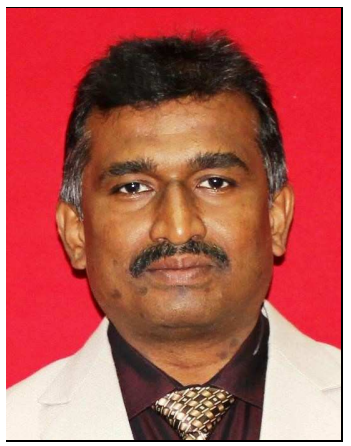

Gowrishankar $\mathbf{S}$ is a senior Professor at Computer Science \& Engineering department at BMS College of Engineering, Bangalore. He served as a Head of the department of CS\&E and IS\&E of BMSCE. He is actively associated with the Research Collaborative Sabbatical program with University of Alabama, Huntsville (UAH), USA and he is a visiting professor for UAH. Having an Academic and Research experience of 20 years, he authored more than 80 research publications in reputed international journals and conferences. His research interests include performance evaluation, wireless network and deep learning.

(i) https://orcid.org/0000-0002-8119-8711

E-mail: Gowrishankar.cse@bmsce.ac.in

B.M.S.C.E

Basavangudi

Bangalore, India 